\title{
ON QUASI-LOCAL NOETHERIAN RINGS
}

\section{GERHARD MICHLER ${ }^{1}$}

It is the purpose of this note to show that each semiprime, quasilocal, noetherian ring with gl. $\operatorname{dim} R \leqq 2$ is Morita equivalent to a quasi-local noetherian domain $D$ with gl. $\operatorname{dim} D \leqq 2$ (cf. Theorem 1$)$.

All rings considered here have an identity element; all modules are assumed to be unitary. The ring $R$ is noetherian if $R$ satisfies the ascending chain conditions for right and for left ideals. A domain is a ring without zero-divisors $z \neq 0$. The ring $R$ is quasi-local, if its Jacobson radical $J$ is its unique maximal two-sided ideal.

Our result here is another consequence of the Morita Theorems (cf. Auslander and Goldman [1, Appendix]). In order to apply them we need the following standard notation:

If $P$ is a finitely generated right $R$-module, and $T=\operatorname{End}_{k}(P)$, then $P$ is also a left $T$-module. The map

$$
\tau: \operatorname{Hom}_{R}(P, R) \otimes_{T} P \rightarrow R
$$

which is defined by $\tau(f \otimes x)=f(x)$ for all $x \in P$ and all $f \in \operatorname{Hom}_{R}(P, R)$ is called the trace mapping of the $R$-module $P$. The image $\tau_{R}(P)$ of $\tau$ is the trace ideal of $P$. One statement of the Morita Theorems is that $\tau_{R}(P)$ is an idempotent, two-sided ideal of $R$, if $P$ is a finitely generated projective right $R$-module. In case we also have $\tau_{R}(P)=R$, then $P$ is a finitely generated projective left $T$-module, and $R \cong \operatorname{End}_{\mathfrak{J}}(P)$.

Theorem 1. The ring $R$ is a semiprime, quasi-local, noetherian ring with gl. $\operatorname{dim} R \leqq 2$ if and only if $R$ is isomorphic to the full ring of endomorphisms $\operatorname{End}_{D}(P)$ of a finitely generated projective left $D$-module $P$ over a quasi-local, noetherian domain with gl. $\operatorname{dim} D \leqq 2$.

Proor. If $R$ is a semiprime noetherian ring, then $R$ has a uniform right annihilator $P \neq 0$ by Goldie [2, p. 205, Theorem 2.3]. Hence $P=t_{r}=\{x \in R \mid t x=0\}$ for some $0 \neq t \in R$ by Goldie [2, p. 208, Theorem 3.7]. Since gl. $\operatorname{dim} R \leqq 2$, the following standard exact sequence

$$
0 \leftarrow R / t R \leftarrow R \nwarrow_{t R} \swarrow^{R} \nwarrow_{t_{r}}=P^{\swarrow} \swarrow^{0}
$$

shows that $P$ is a projective right $R$-module, which is finitely gen-

Received by the editors August 7, 1967.

${ }^{1}$ Partially supported by National Science Foundation grant GP 6419. 
erated. Thus $0 \neq \tau_{R}(P)=S$ is an idempotent ideal of $R$. Since $R$ is quasi-local, either $S \leqq J$ or $S=R$ where $J$ is the Jacobson radical of $R$. Therefore $S=S J=0$ by Nakayama's Lemma, in case $S \leqq J$. This implies $S=R$, and so $R \cong \operatorname{End}_{D}(P)$ by the above remarks, where $D=\operatorname{End}_{R}(P)$. Furthermore, $P$ is a finitely generated projective left $D$-module. If the right $R$-module $P$ is generated by $n$ elements, then $P$ is a direct summand of a free right $R$-module $F$ on $n$ generators. Let $B=\operatorname{End}_{D}(F)$. Then there is an idempotent $0 \neq e \in B$ such that $D \cong e B e$. Clearly, $B$ is quasi-local and noetherian, and $\mathrm{gl}$. $\operatorname{dim} B \leqq 2$ by Harada [3, Theorem 2]. Hence $B$ e $B=B$, which implies again by the Morita Theorems that $e B$ is a projective left $e B e$-module. Thus gl. $\operatorname{dim} D=$ gl. $\operatorname{dim}(e B e) \leqq$ gl. $\operatorname{dim} B \leqq 2$ by Harada $[3$, p. 27, Theorem 8]. Obviously $D$ is quasi-local and noetherian. Since $D$ is the full ring of $R$-endomorphisms of the uniform right ideal $P$ of $R, D$ is a domain by Goldie $[2$, p. 218 , Theorem 5.6]. This completes the proof of Theorem 1, because the converse part is now obvious.

CoRollary 1. A semiprime, quasi-local, noetherian ring $R$ with gl. $\operatorname{dim} R \leqq 2$ is a prime ring.

The proof follows at once from Theorem 1 , because it states that $R$ is Morita equivalent to a domain.

REMARK. We do not know whether Theorem 1 holds, if we drop the requirement that $R$ be semiprime, but assume that $R$ has an artinian total ring of quotients. Since hereditary, quasi-local, noetherian rings are prime rings, one could expect an affirmative answer to this question. For quasi-local noetherian rings with gl. $\operatorname{dim} R \leqq 2$ whose Jacobson radical is a principal right ideal we can show that they are prime rings, because the following statement holds.

COROLlARY 2. If $R$ is a quasi-local noetherian ring with $\mathrm{gl}$. $\operatorname{dim} R \leqq 2$ whose Jacobson radical is a principal right ideal of $R$, then $R$ is Morita equivalent to either a simple noetherian domain or to a quasi-local noetherian domain $D$ with $\mathrm{gl}$. $\operatorname{dim} D \leqq 2$.

Proof. Since $R$ is a quasi-local noetherian ring whose Jacobson radical $J$ is a principal right ideal of $R$, the ring $R$ is either semiprime or $J$ is nilpotent by [4, Hilfssatz 4.1]. By Theorem 1 we may assume that $J$ is nilpotent. If $J=n R$, then let $P=n_{l}=\{x \in R \mid x n=0\}$. Since $g l . \operatorname{dim} R \leqq 2, P$ is a finitely generated projective left $R$-module. Therefore $\tau_{R}(P)=R$, because $R$ is quasi-local. Hence

$$
1=p_{1} f_{1}+p_{2} f_{2}+\cdots+p_{n} f_{n}
$$

for some $p_{i} \in P$ and some $f_{i} \in \operatorname{Hom}_{R}(P, R)$. If we had $P \leqq J$, then there 
would be a smallest positive integer $k$ such that $P^{k}=0$. Since $k \neq 1$, there exists $0 \neq y \in P^{k-1}$. Now

$$
\begin{aligned}
y & =y\left(p_{1} f_{1}\right)+y\left(p_{2} f_{2}\right)+\cdots+y\left(p_{n} f_{n}\right) \\
& =\left(y p_{1}\right) f_{1}+\left(y p_{2}\right) f_{2}+\cdots+\left(y p_{n}\right) f_{n}=0,
\end{aligned}
$$

because $y p_{i} \in P^{k}=0$ for all $i$. This contradiction shows $P \leq J$. Hence $R=P R$ by Nakayama's Lemma. Thus

$$
R n=P R n \leqq P(n R)=(P n) R=0 .
$$

Therefore $J=0$, which implies that $R$ is simple. Hence $R$ is Morita equivalent to a simple noetherian domain $D$ with $\mathrm{gl}$. $\operatorname{dim} R \leqq 2$ by Theorem 2, completing the proof of Corollary 2.

\section{REFERENCES}

1. M. Auslander and O. Goldman, Maximal orders, Trans. Amer. Math. Soc. 97 (1960), 1-24.

2. A. W. Goldie, Semi-prime rings with maximum condition, Proc. London Math. Soc. (3) 10 (1960), 201-220.

3. M. Harada, Note on the dimension of modules and algebras, J. Inst. Polyteck. Osaka City Univ. 7 (1956), 17-27.

4. G. Michler, Charakterisierung einer Klasse von Noetherschen Ringen, Math. Z. 100 (1967), 163-182.

Cornell University 